\title{
HAT-P-17b,c: A TRANSITING, ECCENTRIC, HOT SATURN AND A LONG-PERIOD, COLD JUPITER*
}

\author{
A. W. Howard ${ }^{1,11}$, G. Á. Bakos ${ }^{2,12}$, J. Hartman ${ }^{2}$, G. Torres ${ }^{2}$, A. ShPorer ${ }^{3,4}$, T. Mazeh ${ }^{5}$ GéZa Kovács $^{6}$, D. W. Latham $^{2}$, \\ R. W. Noyes ${ }^{2}$, D. A. Fischer ${ }^{7}$, J. A. Johnson ${ }^{8}$, G. W. MArcy ${ }^{1}$, G. A. Esquerdo ${ }^{2}$, B. Béky ${ }^{2}$, R. P. Butler ${ }^{9}$, D. D. SAsselov ${ }^{2}$, \\ R. P. Stefanik ${ }^{2}$, G. Perumpilly ${ }^{2}$, J. LÁzÁR ${ }^{10}$, I. PAPP ${ }^{10}$, AND P. SÁRI ${ }^{10}$ \\ ${ }^{1}$ Department of Astronomy, University of California, Berkeley, CA, USA; howard@ astro.berkeley.edu \\ ${ }^{2}$ Harvard-Smithsonian Center for Astrophysics, Cambridge, MA, USA; gbakos@cfa.harvard.edu \\ ${ }^{3}$ LCOGT, 6740 Cortona Drive, Santa Barbara, CA, USA \\ ${ }^{4}$ Department of Physics, Broida Hall, UC Santa Barbara, CA, USA \\ ${ }^{5}$ School of Physics and Astronomy, Raymond and Beverly Sackler Faculty of Exact Sciences, Tel Aviv University, Tel Aviv 69978, Israel \\ ${ }^{6}$ Konkoly Observatory, Budapest, Hungary \\ ${ }^{7}$ Astronomy Department, Yale University, New Haven, CT, USA \\ ${ }^{8}$ California Institute of Technology, Department of Astrophysics, MC 249-17, Pasadena, CA, USA \\ ${ }^{9}$ Department of Terrestrial Magnetism, Carnegie Institute of Washington, DC, USA \\ ${ }^{10}$ Hungarian Astronomical Association, Budapest, Hungary \\ Received 2010 August 24; accepted 2011 August 23; published 2012 April 2
}

\begin{abstract}
We report the discovery of HAT-P-17b,c, a multi-planet system with an inner transiting planet in a short-period, eccentric orbit and an outer planet in a $4.4 \mathrm{yr}$, nearly circular orbit. The inner planet, HAT-P-17b, transits the bright $V=10.54$ early K dwarf star GSC 2717-00417, with an orbital period $P=10.338523 \pm 0.000009$ days, orbital eccentricity $e=0.342 \pm 0.006$, transit epoch $T_{c}=2454801.16943 \pm 0.00020$ (BJD: barycentric Julian dates throughout the paper are calculated from Coordinated Universal Time (UTC)), and transit duration $0.1690 \pm 0.0009$ days. HAT-P-17b has a mass of $0.534 \pm 0.018 M_{\mathrm{J}}$ and radius of $1.010 \pm 0.029 R_{\mathrm{J}}$ yielding a mean density of $0.64 \pm 0.05 \mathrm{~g} \mathrm{~cm}^{-3}$. This planet has a relatively low equilibrium temperature in the range $780-927 \mathrm{~K}$, making it an attractive target for follow-up spectroscopic studies. The outer planet, HAT-P-17c, has a significantly longer orbital period $P_{2}=1610 \pm 20$ days and a minimum mass $m_{2} \sin i_{2}=1.31_{-0.15}^{+0.18} M_{\mathrm{J}}$. The orbital inclination of HAT-P-17c is unknown as transits have not been observed and may not be present. The host star has a mass of $0.86 \pm 0.04 M_{\odot}$, radius of $0.84 \pm 0.02 R_{\odot}$, effective temperature $5246 \pm 80 \mathrm{~K}$, and metallicity $[\mathrm{Fe} / \mathrm{H}]=0.00 \pm$ 0.08 . HAT-P-17 is the second multi-planet system detected from ground-based transit surveys.
\end{abstract}

Key words: planetary systems - stars: individual (HAT-P-17, GSC 2717-00417) - techniques: photometric techniques: spectroscopic

Online-only material: color figure, machine-readable table

\section{INTRODUCTION}

With nearly 100 confirmed transiting extrasolar planets (TEPs) known, many studies of planetary properties now focus on the statistical distributions of and correlations between planetary parameters. Individual TEPs still remain extraordinarily valuable, particularly if they have properties that exemplify an important subgroup of planets and orbit stars that are bright enough for meaningful follow-up observations. Such iconic well-studied planets include HD 209458b (Charbonneau et al. 2000; Henry et al. 2000), HD 189733 (Bouchy et al. 2005), GJ 436b (Gillon et al. 2007; Butler et al. 2004), HAT-P-13b,c (Bakos et al. 2010a), WASP-12b (Hebb et al. 2009), and GJ 1214b (Charbonneau et al. 2009). The HAT-P-17 system has at least two unusual properties compared to the ensemble of known TEPs and may serve as an exemplar for planets with these properties. The atmosphere of HAT-P-17b is relatively cool for a TEP and HAT-P-17c is one of only two long-period planets found to orbit a TEP host.

The distribution of TEPs discovered by ground-based transit surveys is biased toward large planets orbiting faint early-type

\footnotetext{
* Based in part on observations obtained at the W. M. Keck Observatory, which is operated by the University of California and the California Institute of Technology. Keck time has been granted by NOAO and NASA.

${ }^{11}$ Townes Postdoctoral Fellow.

12 NSF Fellow.
}

stars in short-period orbits. Each of these biases stems from the observational selection effects of the surveys that have detected the majority of TEPs: the deep transits of large planets are easier to detect; early-type stars dominate magnitude-limited surveys; and short-period orbits have higher a priori transit probabilities and significantly larger probabilities of detection in a ground-based survey limited to one or two observing sites. The overabundance of detected short-period planets has distorted our perception of the atmospheric properties of extrasolar jovian planets. Planets in $P \sim 3$ day orbits $(a \sim 0.04)$ experience intense interactions with the radiation and tides of their host stars. The atmospheres of many of these planets are inflated beyond the radii predicted by theoretical models (e.g., Fortney et al. 2007). To understand cooler planets, which we know from radial velocity (RV) surveys represent the vast majority of gas giants (Wright et al. 2009), we must study planets orbiting progressively further from their host stars.

With such a scarcity of transiting cool planets, HAT-P-17b is a valuable probe of the planetary mass-radius relationship and additional properties through follow-up observations. Together, the relatively long orbital period and later spectral type of the host star yield an incident stellar flux that is one to two orders of magnitude lower than the flux received by most detected TEPs (Kovács et al. 2010). The host star is also relatively bright $(V=10.54)$ making follow-up atmospheric studies conceivable. While the Kepler mission (Borucki et al. 2011) 
has been extraordinarily successful in the detection of TEPs, the vast majority of its discovered planets orbit faint stars; only $1.5 \%$ of the $\sim 10^{5}$ stars being surveyed (Batalha et al. 2010) are brighter than 11th magnitude (Kepler magnitude). We predict that HAT-P-17b will be among the small number of well-studied cooler $\left(T_{\text {eff }}<1000 \mathrm{~K}\right)$ TEPs.

Prior to this announcement, only one TEP discovered by a ground-based photometric survey is in a confirmed multiplanet system. HAT-P-13 has a hot Jupiter inner planet and highly eccentric super-Jupiter outer planet with an orbital period of 450 days (Bakos et al. 2010a). The outer planet, HAT-P-13c, was detected only in RV measurements and has not been shown to transit. The system reported here, HAT-P-17, is now the second TEP discovered by a ground-based transit survey with a confirmed second planet. The relatively low rate of detected planet multiplicity among TEPs discovered from the ground may be skewed by the lack of long-term RV and photometric monitoring for most TEP host stars. Measuring the rate of planet multiplicity among stars hosting a hot Jupiter will probe the dynamical histories and migration mechanisms of hot Jupiters (see, e.g., Wu et al. 2007). Multi-planet systems are significantly more common among RV-detected systems; Wright et al. (2009) find that $28 \%$ of known planet host stars are multi-planet systems.

Several multi-planet systems have also been discovered from space. Corot-7 is thought to host two short-period super-Earths (Léger et al. 2009; Queloz et al. 2009), one of which transits. The Kepler mission recently announced five candidate systems with multiple transiting planets and is poised to announce additional systems (Steffen et al. 2010).

The Hungarian-made Automated Telescope Network (HATNet; Bakos et al. 2004) survey has been one of the main contributors to the discovery of TEPs. In operation since 2003, it has now covered approximately $14 \%$ of the sky, searching for TEPs around bright stars $(8 \lesssim I \lesssim 14.0)$. HATNet operates six wide-field instruments: four at the Fred Lawrence Whipple Observatory (FLWO) in Arizona, and two on the roof of the hangar servicing the Smithsonian Astrophysical Observatory's Submillimeter Array, in Hawaii. Since 2006, HATNet has announced and published 16 TEPs. In this work, we report our 17th discovery, around the relatively bright star previously known as GSC 2717-00417.

The layout of the paper is as follows. In Section 2, we report the detection of the photometric signal and the followup spectroscopic and photometric observations of HAT-P-17. In Section 3, we describe the analysis of the data, beginning with the determination of the stellar parameters, continuing with a discussion of the methods used to rule out nonplanetary, false positive scenarios which could mimic the photometric and spectroscopic observations, and finishing with a description of our global modeling of the photometry and RVs. In Section 4, we discuss implications of this discovery, compare our results with recent theoretical models of TEPs, and consider possible follow-on observations.

\section{OBSERVATIONS}

\subsection{Photometric Detection}

The transits of HAT-P-17b were detected with the HAT-5 telescope in Arizona, the HAT-8 telescope in Hawaii, and with the Wise-HAT (WHAT) telescope at Wise Observatory in Israel (Shporer et al. 2009). The region around GSC 2717-00417, a field internally labeled as 247 , was observed on a nightly basis between 2005 May 8 and 2005 October 24, whenever weather conditions permitted. We gathered 9686 exposures of 5 minutes duration at a 5.5 minute cadence. Each image contained approximately 85,000 stars down to $I \sim 14.0$. For the brightest stars in the field, we achieved a per-image photometric precision of $5 \mathrm{mmag}$. The star is also located in the overlapping field 248, which was observed with the HAT-6 telescope in Arizona and the WHAT telescope in Israel between 2004 June 4 and 2004 November 10, and between 2005 July 3 and 2005 July 15. Altogether 4882 exposures of 5 minutes duration at 5.5 minute cadence were gathered for this field.

The calibration of the HATNet and WHAT frames was carried out using standard photometric procedures. The calibrated images were then subjected to star detection and astrometry, as described in Pál \& Bakos (2006). Aperture photometry was performed on each image at the stellar centroids derived from the Two Micron All Sky Survey (2MASS; Skrutskie et al. 2006) catalog and the individual astrometric solutions. The resulting light curves were decorrelated (cleaned of trends) using the external parameter decorrelation (EPD; see Bakos et al. 2010b) technique in "constant" mode and the trend filtering algorithm (TFA; see Kovács et al. 2005). The light curves were searched for periodic box-shaped signals using the box leastsquares (BLS; see Kovács et al. 2002) method. We detected a significant signal in the light curve of GSC 2717-00417 (also known as 2MASS 21380873+3029193 and TYC 2717-417-1; $\alpha=21^{\mathrm{h}} 38^{\mathrm{m}} 08^{\mathrm{s}} .88, \delta=+30^{\circ} 29^{\prime} 19^{\prime} .4 ; \mathrm{J} 2000 ; V=10.54$; Droege et al. 2006), with an apparent depth of $\sim 12.0 \mathrm{mmag}$, and a period of $P=10.3385$ days (see Figure 1). The drop in brightness had a first-to-last-contact duration, relative to the total period, of $q=0.0163 \pm 0.0001$, corresponding to a total duration of $P q=4.057 \pm 0.022 \mathrm{hr}$ (see Figure 1). We note that the transits were only detected from the observations of field 247, and were not detected in the observations of field 248.

\subsection{Reconnaissance Spectroscopy}

As is routine in the HATNet project, all candidates are subjected to careful scrutiny before investing valuable time on large telescopes. This includes spectroscopic observations at relatively modest facilities to establish whether the transitlike feature in the light curve of a candidate might be due to astrophysical phenomena other than a planet transiting a star. Many of these false positives are associated with large RV variations in the star (tens of $\mathrm{km} \mathrm{s}^{-1}$ ) that are easily recognized.

One of the tools we have used for this purpose is the HarvardSmithsonian Center for Astrophysics (CfA) Digital Speedometer (DS; Latham 1992), an echelle spectrograph mounted on the FLWO $1.5 \mathrm{~m}$ telescope. This instrument delivers high-resolution spectra $(\lambda / \Delta \lambda \approx 35,000)$ over a single order centered on the Mg I b triplet ( $\sim 5187 \AA$ ), with typically low signal-to-noise ratios (S/Ns) that are nevertheless sufficient to derive RVs with moderate precisions of $0.5-1.0 \mathrm{~km} \mathrm{~s}^{-1}$ for slowly rotating stars. The same spectra can be used to estimate the effective temperature, surface gravity, and projected rotational velocity of the host star, as described by Torres et al. (2002). With this facility, we are able to reject many types of false positives, such as F dwarfs orbited by $\mathrm{M}$ dwarfs, grazing eclipsing binaries, or triple or quadruple star systems. Additional tests are performed with other spectroscopic observations described in the next section.

For HAT-P-17, we obtained eight observations with the DS between September and November of 2007. The velocity measurements showed an rms residual of $0.60 \mathrm{~km} \mathrm{~s}^{-1}$, consistent with no detectable RV variation within the precision of the 

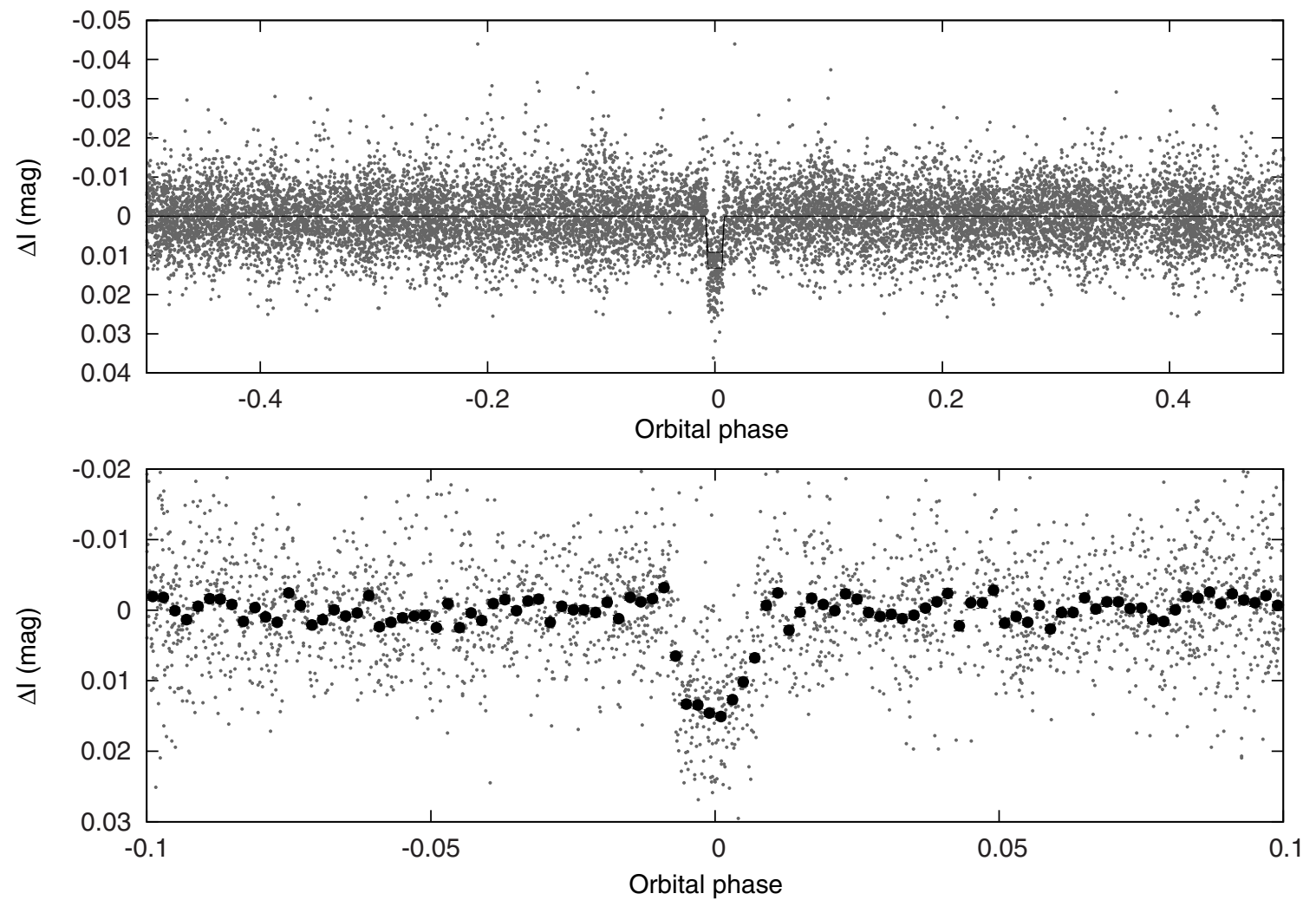

Figure 1. Unbinned (top) and binned (bottom) light curves of HAT-P-17 including all 14,000 instrumental $I$-band 5.5 minute cadence measurements obtained with the HAT-5, HAT-6, and HAT-8 telescopes of HATNet and with the WHAT telescope (see the text for details), and folded with the period $P=10.3385227$ days (resulting from the global fit described in Section 3). The solid line shows the "P1P3" transit model fit to the light curve (Section 3.3).

measurements. All spectra were single lined, i.e., there is no evidence for additional stars in the system. The atmospheric parameters we infer from these observations are the following: effective temperature $T_{\mathrm{eff} \star}=5250 \pm 125 \mathrm{~K}$, surface gravity $\log g_{\star}=4.5 \pm 0.25(\log \mathrm{cgs})$, and projected rotational velocity $v \sin i=0.0_{-0.0}^{+0.5} \mathrm{~km} \mathrm{~s}^{-1}$. The effective temperature corresponds to an early $\mathrm{K}$ dwarf star. The mean heliocentric RV of HAT-P-17 is $\gamma_{\mathrm{RV}}=20.13 \pm 0.21 \mathrm{~km} \mathrm{~s}^{-1}$.

\subsection{High-resolution, High-S/N Spectroscopy}

Given the significant transit detection by HATNet, and the encouraging DS results that rule out obvious false positives, we proceeded with the follow-up of this candidate by obtaining high-resolution, high-S/N spectra to characterize the RV variations, and to refine the determination of the stellar parameters. For this we used HIRES (Vogt et al. 1994) on the Keck I telescope located on Mauna Kea, Hawaii, between 2007 October and 2010 April. The width of the spectrometer slit was 0 .'86, resulting in a resolving power of $\lambda / \Delta \lambda \approx 55,000$, with a wavelength coverage of $\sim 3800-8000 \AA$.

We obtained 32 HIRES exposures with an iodine cell mounted directly in front of the spectrometer entrance slit. The dense set of molecular absorption lines imprinted on the stellar spectra provides a robust wavelength fiducial against which Doppler shifts are measured, as well as strong constraints on the shape of the spectrometer instrumental profile at the time of each observation (Marcy \& Butler 1992; Valenti et al. 1995). An additional exposure was taken without the iodine cell, for use as a template in the reductions. Relative RVs in the solar system barycentric frame were derived as described by Butler et al. (1996), incorporating full modeling of the spatial and temporal variations of the instrumental profile. These measurements have typical uncertainties of $1.5-2.0 \mathrm{~m} \mathrm{~s}^{-1}$ for spectra with per-pixel S/Ns of 100-150. HIRES measurements of late G and early $\mathrm{K}$ dwarf stars have achieved long-term stability of $1.5-2.0 \mathrm{~m} \mathrm{~s}^{-1}$ on standard stars, including noise from systematic and astrophysical sources (Howard et al. 2011b). The RV measurements and their uncertainties are listed in Table 1. The period-folded data, along with our best fit described below in Section 3, are displayed in Figure 2.

In the same figure we also show the relative $S$ index, which is a measure of the chromospheric activity of the star derived from the flux in the cores of the $\mathrm{Ca}$ II $\mathrm{H}$ and $\mathrm{K}$ lines. This index was computed following the prescription given by Vaughan et al. (1978), after matching each spectrum to a reference spectrum using a transformation that includes a wavelength shift and a flux scaling that is a polynomial as a function of wavelength. The transformation was determined on regions of the spectra that are not used in computing this indicator. Note that our relative $S$ index has not been calibrated to the scale of Vaughan et al. (1978). We do not detect any significant variation of the index correlated with the orbital phase of either planet; such a correlation might have indicated that the RV variations could be due to stellar activity, casting doubt on the planetary nature of the candidates.

In addition, we computed an $S_{\mathrm{HK}}$ index calibrated to the Mt. Wilson scale, permitting comparisons with calibrated activity measurements of other stars (Knutson et al. 2010). We find $S_{\mathrm{HK}}=0.162$ (median of all HIRES measurements) and $\log R_{\mathrm{HK}}^{\prime}=-5.039$ (median). These measurements employ the techniques described in Isaacson \& Fischer (2010), calibrated on 1500 stars observed by the California Planet Survey (CPS). We used $B-V=0.83$ estimated from $T_{\text {eff }}$ using the linear transformation between those quantities in Valenti \& Fischer (2005). 

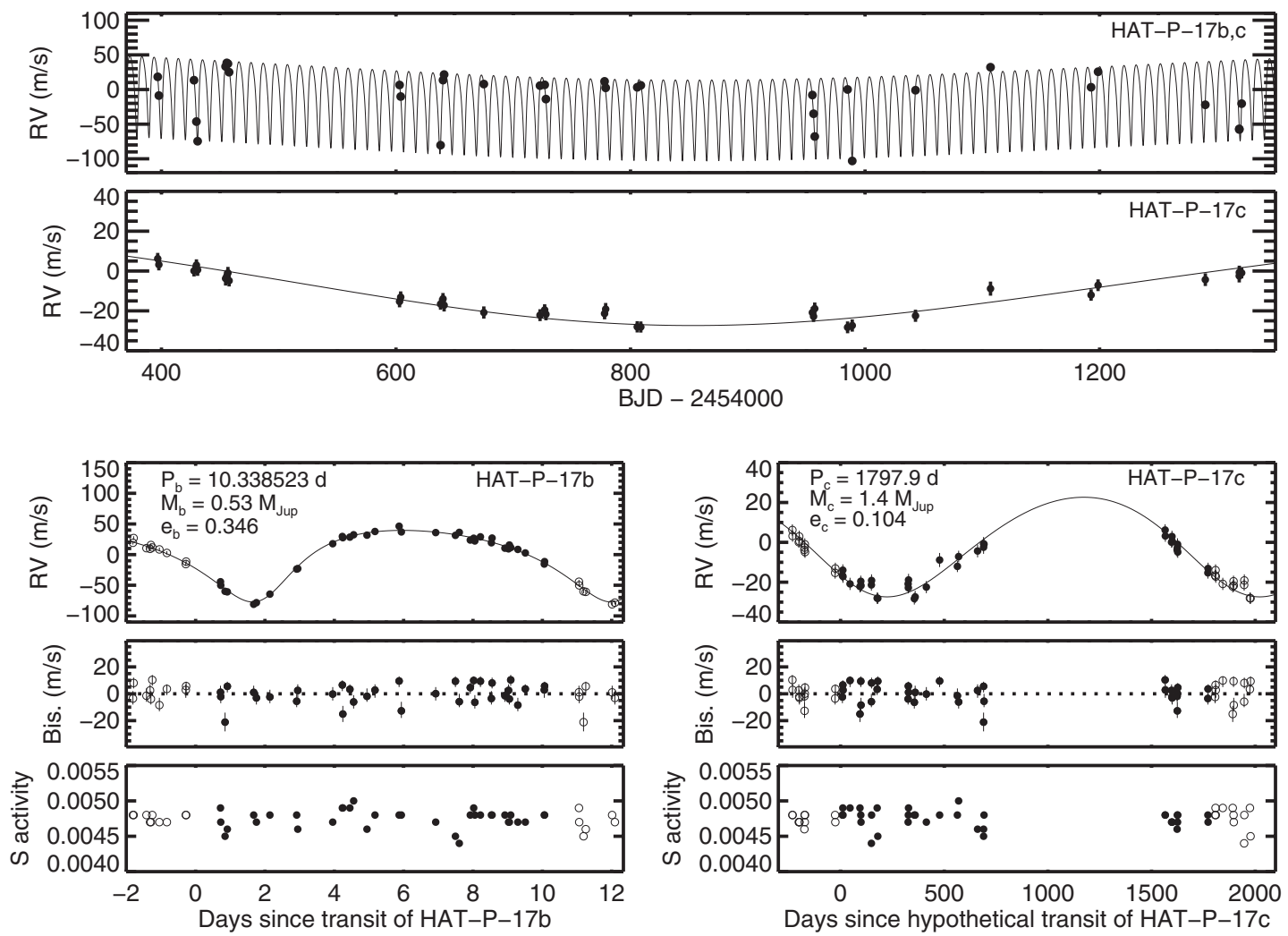

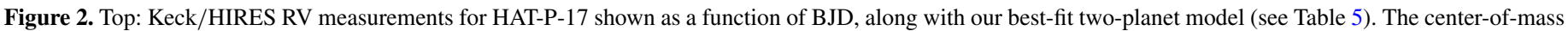

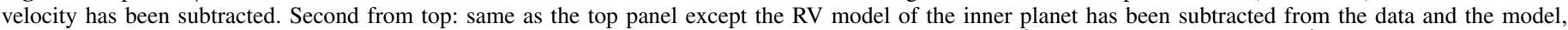

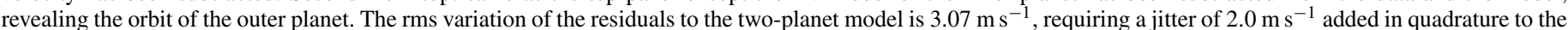

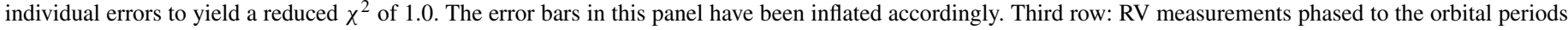

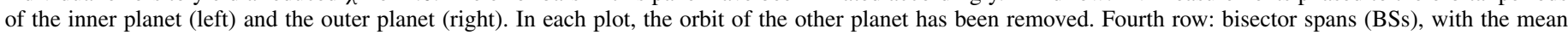

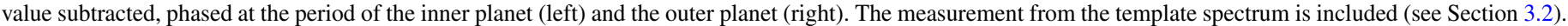

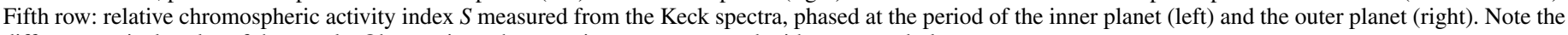
different vertical scales of the panels. Observations shown twice are represented with open symbols.

\subsection{Photometric Follow-up Observations}

In order to permit a more accurate modeling of the light curve, we conducted additional photometric observations using a variety of facilities, including: the KeplerCam CCD camera on the FLWO $1.2 \mathrm{~m}$ telescope, the $0.6 \mathrm{~m}$ Schmidt telescope of Konkoly Observatory at the Piszkéstetô Mountain Station, and the $0.46 \mathrm{~m}$ and $1.0 \mathrm{~m}$ telescopes at Wise Observatory. We observed three transit events of HAT-P-17 with the FLWO $1.2 \mathrm{~m}$ telescope on the nights of 2007 December 14, 2008 October 19, and 2009 October 16, while the event on 2008 September 8 was observed simultaneously at Konkoly Observatory and with the two telescopes at Wise Observatory (Figure 3). These observations are summarized in Table 2.

The reduction of these images, including basic calibration, astrometry, and aperture photometry, was performed as described by Bakos et al. (2010b). We performed EPD and TFA to remove trends simultaneously with the light curve modeling (see Section 3, and Bakos et al. (2010b) for details). The final time series are shown in the top portion of Figure 3, along with our best-fit transit light curve model described below; the individual measurements are reported in Table 3.

\section{ANALYSIS}

\subsection{Properties of the Parent Star}

Fundamental parameters of the host star HAT-P-17 such as the mass $\left(M_{\star}\right)$ and radius $\left(R_{\star}\right)$, which are needed to infer the planetary properties, depend strongly on other stellar quantities that can be derived spectroscopically. For this we have relied on the HIRES template spectrum, and the analysis package known as Spectroscopy Made Easy (SME; Valenti \& Piskunov 1996), along with the atomic line database of Valenti \& Fischer (2005). SME yielded the following initial values and uncertainties (which we have conservatively increased to include our estimates of the systematic errors): effective temperature $T_{\text {eff }}=$ $5455 \pm 80 \mathrm{~K}$, stellar surface gravity $\log g_{\star}=4.60 \pm 0.10$ (cgs), metallicity $[\mathrm{Fe} / \mathrm{H}]=+0.13 \pm 0.08 \mathrm{dex}$, and projected rotational velocity $v \sin i=0.5 \pm 0.5 \mathrm{~km} \mathrm{~s}^{-1}$. We adopt the single-sided uncertainty of $\pm 0.5 \mathrm{~km} \mathrm{~s}^{-1}$ on $v \sin i$ from Valenti \& Fischer (2005) based on their SME analysis of nearly 2000 stars. For this star and others with low $v \sin i$, the true error distribution excludes unphysical values $\left(v \sin i<0 \mathrm{~km} \mathrm{~s}^{-1}\right)$ and is likely asymmetric.

In principle, the effective temperature and metallicity, along with the surface gravity taken as a luminosity indicator, could be used as constraints to infer the stellar mass and radius by comparison with stellar evolution models. However, the effect of $\log g_{\star}$ on the spectral line shapes is subtle, and as a result it is typically difficult to determine accurately, so that in practice it is a poor luminosity indicator. For planetary transits, a stronger constraint is often provided by $a / R_{\star}$, the normalized semimajor axis, which is closely related to $\rho_{\star}$, the mean stellar density. The quantity $a / R_{\star}$ can be derived directly from the transit light curves (see Sozzetti et al. 2007, and also Section 3.3). This, in turn, improves our determination of the 


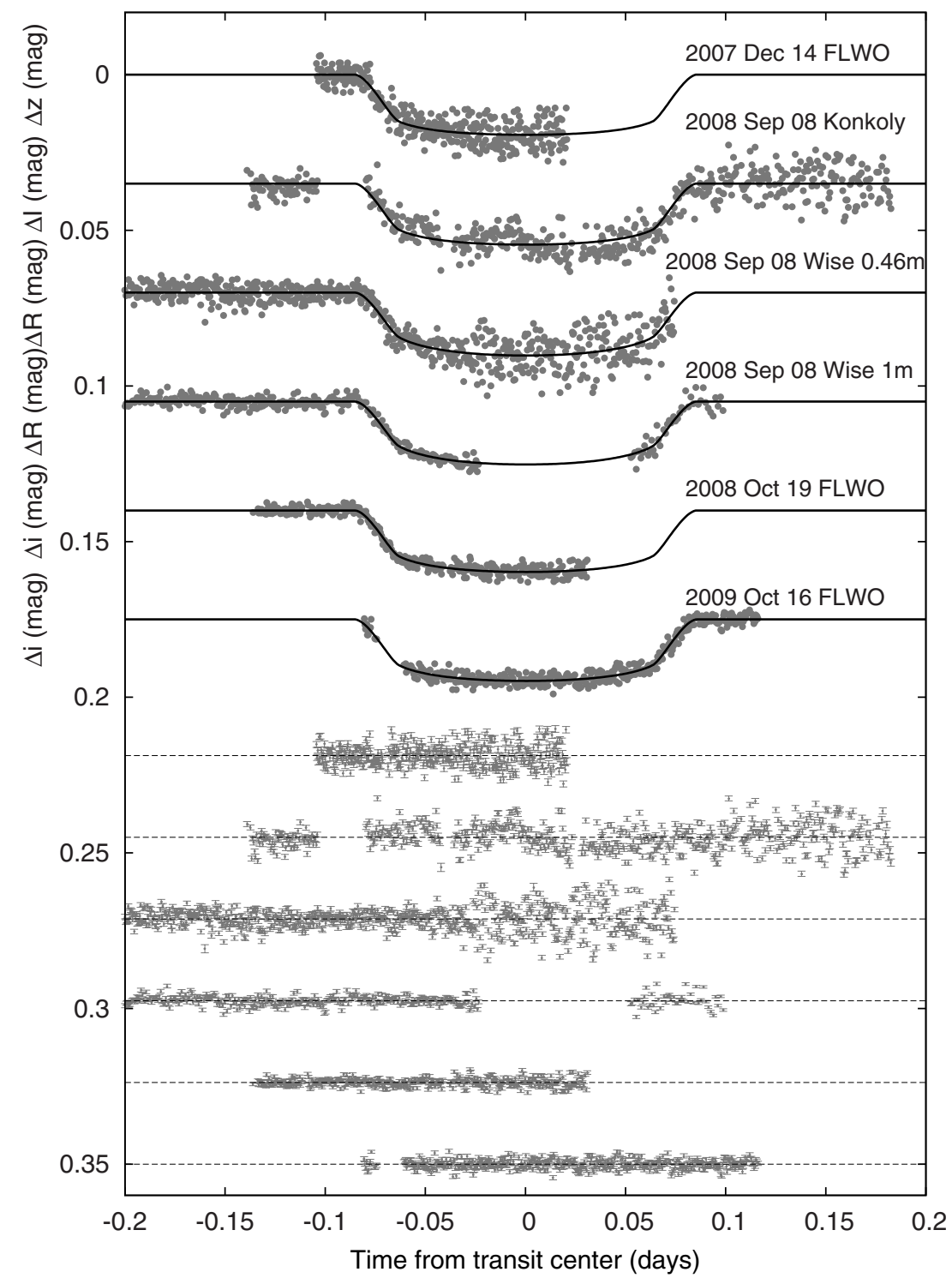

Figure 3. Unbinned instrumental $z$-band, $I$-band, $R$-band, and $i$-band transit light curves, acquired with KeplerCam at the FLWO $1.2 \mathrm{~m}$ telescope, with the Schmidt telescope at Konkoly Observatory, and with the $0.46 \mathrm{~m}$ and $1 \mathrm{~m}$ telescopes at Wise Observatory. The light curves have been EPD- and TFA-processed, as described in Section 3.3. The dates and facilities used to observe the events are indicated. Curves after the first are displaced vertically for clarity. Our best fit from the global modeling described in Section 3.3 is shown by the solid lines. Residuals from the fits are displayed at the bottom, in the same order as the top curves. The error bars represent photon and background shot noise, plus readout noise.

spectroscopic parameters by supplying an indirect constraint on the weakly determined spectroscopic value of $\log g_{\star}$ that removes degeneracies. We take this approach here, as described below. The validity of our assumption, namely, that the adequate physical model describing our data is a planetary transit (as opposed to a blend), is shown later in Section 3.2.

Our initial values of $T_{\text {eff }}, \log g_{\star}$, and $[\mathrm{Fe} / \mathrm{H}]$ were used to determine auxiliary quantities needed in the global modeling of the follow-up photometry and RVs (specifically, the limbdarkening coefficients). This modeling, the details of which are described in Section 3.3, uses a Monte Carlo approach to deliver the probability distribution of $a / R_{\star}$ and other fitted variables. See Pál (2009b) for further details. When combining $a / R_{\star}$ (a luminosity proxy) with assumed Gaussian distributions for $T_{\text {eff }}$ and $[\mathrm{Fe} / \mathrm{H}]$ from SME, we compare with stellar evolution models to estimate the probability distributions of additional inferred stellar parameters, including $\log g_{\star}$. Here, we use the stellar evolution calculations from the Yonsei-Yale (YY) series by Yi et al. (2001). The comparison with the model isochrones was carried out for each of 20,000 Monte Carlo trial sets (see Section 3.3). Parameter combinations corresponding to unphysical locations in the $\mathrm{H}-\mathrm{R}$ diagram (1.5\% of the trials) were ignored, and replaced with another randomly drawn parameter set. The result and error estimate for the surface gravity, $\log g_{\star}=4.52 \pm 0.02$, is different from the result of our initial SME analysis, which is not surprising in view of the strong correlations among $T_{\mathrm{eff} \star},[\mathrm{Fe} / \mathrm{H}]$, and $\log g_{\star}$ that are often present in spectroscopic determinations. Therefore, we carried out a second iteration in which we adopted this value of $\log g_{\star}$ and held it fixed in a new SME analysis (coupled with a new global modeling of the RV and light curves), adjusting only $T_{\mathrm{eff} \star},[\mathrm{Fe} / \mathrm{H}]$, and $v \sin i$. This gave $T_{\mathrm{eff} \star}=5246 \pm 80 \mathrm{~K}$, $[\mathrm{Fe} / \mathrm{H}]=0.00 \pm 0.08$, and $v \sin i=0.3 \pm 0.5 \mathrm{~km} \mathrm{~s}^{-1}$, in which the conservative uncertainties for the first two have been increased by a factor of two over their formal values, as before. A further iteration did not change $\log g_{\star}$ significantly, 
Table 1

Relative Radial Velocities, Bisector Spans, and Activity Index Measurements of HAT-P-17

\begin{tabular}{|c|c|c|c|c|c|c|}
\hline $\begin{array}{l}\text { BJD } \\
(2,454,000+)\end{array}$ & $\begin{array}{c}\mathrm{RV}^{\mathrm{a}} \\
\left(\mathrm{m} \mathrm{s}^{-1}\right)\end{array}$ & $\begin{array}{c}\sigma_{\mathrm{RV}}^{\mathrm{b}} \\
\left(\mathrm{m} \mathrm{s}^{-1}\right)\end{array}$ & $\begin{array}{c}\mathrm{BS} \\
\left(\mathrm{ms}^{-1}\right)\end{array}$ & $\begin{array}{c}\sigma_{\mathrm{BS}} \\
\left(\mathrm{m} \mathrm{s}^{-1}\right)\end{array}$ & $S^{\mathrm{c}}$ & $\sigma_{\mathrm{S}}$ \\
\hline 396.82931 & 18.12 & 1.57 & 10.31 & 4.38 & 0.0048 & 0.00002 \\
\hline 397.79661 & -9.05 & 1.67 & 2.84 & 5.15 & 0.0048 & 0.00002 \\
\hline 427.78145 & 13.53 & 1.42 & 2.49 & 5.17 & 0.0047 & 0.00003 \\
\hline 427.78895 & $\ldots$ & $\ldots$ & -2.14 & 5.34 & 0.0047 & 0.00002 \\
\hline 429.81978 & -46.01 & 1.58 & -3.08 & 5.46 & 0.0047 & 0.00003 \\
\hline 430.84830 & -74.24 & 1.78 & -1.88 & 5.52 & 0.0046 & 0.00003 \\
\hline 454.71418 & 32.95 & 2.55 & -12.71 & 6.37 & 0.0048 & 0.00004 \\
\hline 455.70568 & 38.55 & 1.74 & 0.09 & 5.13 & 0.0047 & 0.00002 \\
\hline 456.69684 & 37.13 & 1.84 & 4.61 & 4.86 & 0.0048 & 0.00003 \\
\hline 457.69351 & 25.00 & 1.70 & -3.52 & 5.47 & 0.0048 & 0.00002 \\
\hline 603.04188 & 6.48 & 1.66 & 3.60 & 4.80 & 0.0047 & 0.00002 \\
\hline 604.01999 & -10.27 & 1.67 & -2.39 & 5.19 & 0.0048 & 0.00002 \\
\hline 638.00747 & -80.28 & 1.63 & 6.54 & 4.54 & 0.0049 & 0.00002 \\
\hline 640.09696 & 13.57 & 1.68 & 2.73 & 5.17 & 0.0048 & 0.00002 \\
\hline 641.03845 & 21.59 & 2.09 & 9.89 & 4.28 & 0.0049 & 0.00002 \\
\hline 674.90104 & 7.95 & 1.96 & -15.07 & 6.42 & 0.0049 & 0.00002 \\
\hline 722.81909 & 5.29 & 1.75 & 9.33 & 4.88 & 0.0048 & 0.00002 \\
\hline 726.78740 & 6.67 & 1.54 & -8.43 & 5.62 & 0.0047 & 0.00001 \\
\hline 727.86651 & -14.01 & 1.91 & -5.89 & 5.34 & 0.0044 & 0.00002 \\
\hline 777.87074 & 11.41 & 1.63 & 8.04 & 4.51 & 0.0048 & 0.00002 \\
\hline 778.81597 & 2.54 & 1.98 & 3.34 & 4.82 & 0.0049 & 0.00003 \\
\hline 805.73023 & 3.33 & 1.53 & 9.31 & 4.65 & 0.0045 & 0.00002 \\
\hline 808.77451 & 6.58 & 1.43 & -3.86 & 5.37 & 0.0047 & 0.00002 \\
\hline 955.07012 & -8.12 & 1.43 & 5.73 & 4.54 & 0.0048 & 0.00002 \\
\hline 956.08553 & -35.16 & 1.47 & 1.12 & 5.07 & 0.0049 & 0.00002 \\
\hline 957.07704 & -67.96 & 1.93 & -6.36 & 5.74 & 0.0048 & 0.00002 \\
\hline 985.08988 & 0.22 & 1.74 & 1.03 & 5.04 & 0.0048 & 0.00002 \\
\hline 989.04979 & -103.01 & 1.91 & -0.24 & 5.13 & 0.0047 & 0.00002 \\
\hline 1043.02166 & -0.66 & 1.87 & 9.50 & 4.57 & 0.0048 & 0.00002 \\
\hline 1106.96789 & 32.01 & 2.62 & -1.47 & 5.67 & 0.0048 & 0.00003 \\
\hline 1192.72802 & 3.23 & 1.66 & -6.09 & 5.60 & 0.0050 & 0.00004 \\
\hline 1198.70293 & 25.36 & 1.79 & 2.47 & 5.18 & 0.0046 & 0.00002 \\
\hline 1290.13821 & -21.77 & 2.14 & -21.11 & 7.04 & 0.0045 & 0.00002 \\
\hline 1319.05702 & -56.74 & 2.35 & 5.54 & 4.57 & 0.0046 & 0.00003 \\
\hline 1319.12199 & -57.60 & 2.43 & -5.57 & 5.53 & 0.0048 & 0.00004 \\
\hline 1321.12276 & -20.60 & 1.41 & 1.22 & 5.01 & 0.0047 & 0.00003 \\
\hline 1351.97316 & -26.28 & 1.52 & $\ldots$ & . & $\ldots$ & $\ldots$ \\
\hline 1406.97949 & 52.54 & 1.64 & $\ldots$ & $\ldots$ & $\ldots$ & $\ldots$ \\
\hline 1428.96436 & 50.48 & 2.17 & $\ldots$ & $\ldots$ & $\ldots$ & $\ldots$ \\
\hline 1455.88205 & 12.13 & 1.63 & $\ldots$ & $\ldots$ & . & $\ldots$ \\
\hline 1486.89693 & 13.83 & 1.69 & $\ldots$ & $\ldots$ & $\ldots$ & $\ldots$ \\
\hline 1521.80847 & 56.18 & 1.75 & $\ldots$ & $\ldots$ & $\ldots$ & $\ldots$ \\
\hline 1542.74643 & 56.31 & 1.34 & $\ldots$ & $\ldots$ & $\ldots$ & $\ldots$ \\
\hline
\end{tabular}

Notes. Note that for the iodine-free template exposures we do not measure the RV but do measure the BS and $S$ index. Such template exposures can be distinguished by the missing RV value.

a The zero point of these velocities is arbitrary. An overall offset $\gamma_{\text {rel }}$ fitted to these velocities in Section 3.3 has not been subtracted.

b Internal errors excluding the component of astrophysical jitter considered in Section 3.3.

${ }^{c}$ Relative chromospheric activity index, not calibrated to the scale of Vaughan et al. (1978).

so we adopted the values stated above as the final atmospheric properties of the star. They are collected in Table 4, together with the adopted values for the macroturbulent and microturbulent velocities.

With the adopted spectroscopic parameters, the model isochrones yield the stellar mass and radius, $M_{\star}=0.857 \pm$ $0.039 M_{\odot}$ and $R_{\star}=0.838 \pm 0.021 R_{\odot}$, along with other properties listed at the bottom of Table 4. HAT-P-17 is an early K dwarf

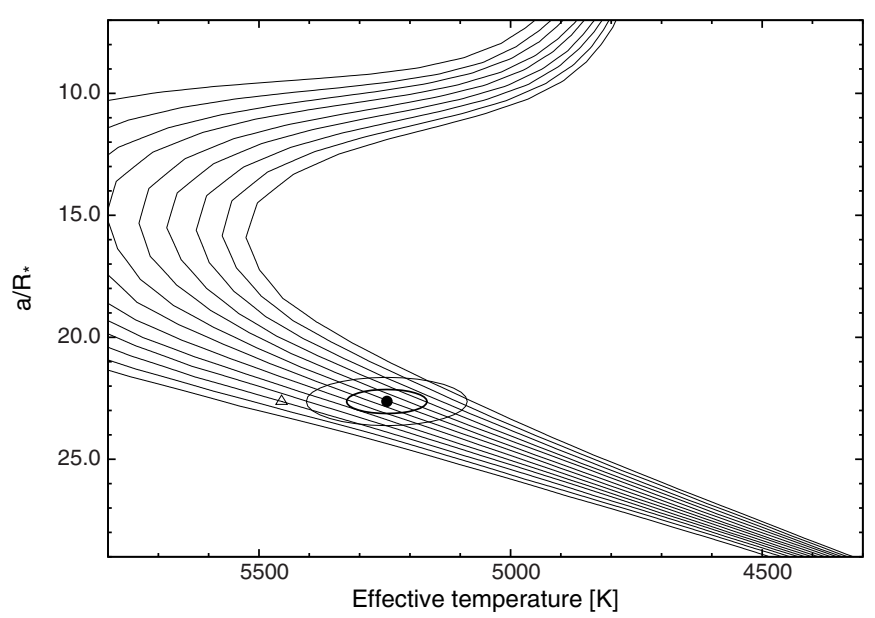

Figure 4. Model isochrones from Yi et al. (2001) for the measured metallicity of HAT-P-17, $[\mathrm{Fe} / \mathrm{H}]=0.00$, and ages between 1.0 and $13.0 \mathrm{Gyr}$ with a step size of $1.0 \mathrm{Gyr}$ (left to right). The adopted values of $T_{\mathrm{eff} \star}$ and $a / R_{\star}$ are shown together with their $1 \sigma$ and $2 \sigma$ confidence ellipsoids. The initial values of $T_{\text {eff }}$ and $a / R_{\star}$ from the first SME and light curve analyses are represented with a triangle.

star with an estimated age of $7.8 \pm 3.3 \mathrm{Gyr}$, according to these models. The inferred location of the star in a diagram of $a / R_{\star}$ versus $T_{\text {eff } \star}$, analogous to the classical H-R diagram, is shown in Figure 4 . The stellar properties and their $1 \sigma$ and $2 \sigma$ confidence ellipsoids are displayed against the backdrop of Yi et al. (2001) isochrones for the measured metallicity of $[\mathrm{Fe} / \mathrm{H}]=0.00$, and a range of ages. For comparison, the location implied by the initial SME results is also shown (triangle).

The stellar evolution modeling provides color indices that may be compared against the measured values as a sanity check. The best available measurements are the near-infrared magnitudes from the 2MASS catalog (Skrutskie et al. 2006), $J_{\text {MASS }}=9.017 \pm 0.022, H_{2 \mathrm{MASS}}=8.619 \pm 0.029$, and $K_{2 \mathrm{MASS}}=8.544 \pm 0.025$; which we have converted to the photometric system of the models (ESO system) using the transformations by Carpenter (2001). The resulting measured color index is $J-K=0.504 \pm 0.036$. This is within $1 \sigma$ of the predicted value from the isochrones of $J-K=0.53 \pm 0.02$. The distance to the object may be computed from the absolute $K$ magnitude from the models $\left(M_{K}=3.79 \pm 0.07\right)$ and the 2MASS $K_{s}$ magnitude, which has the advantage of being less affected by extinction than optical magnitudes. The result is $90 \pm 3 \mathrm{pc}$, where the uncertainty excludes possible systematics in the model isochrones that are difficult to quantify.

\subsection{Spectral Line-bisector Analysis}

Our initial spectroscopic analyses discussed in Sections 2.2 and 2.3 rule out the most obvious astrophysical false positive scenarios. However, more subtle phenomena such as blends (contamination by an unresolved eclipsing binary, whether in the background or associated with the target) can still mimic both the photometric and spectroscopic signatures we see.

Following Torres et al. (2007), we explored the possibility that the measured RVs are not the results of a planet in Keplerian motion, but are instead caused by distortions in the spectral line profiles due to contamination from a nearby unresolved eclipsing binary. A bisector analysis based on the Keck spectra was done as described in Section 5 of Bakos et al. (2007). We detect no variation in excess of the measurement errors in the bisector spans (BSs; see Figure 2). The correlation between the 
Table 2

Summary of Photometric Follow-up Observations

\begin{tabular}{llcrr}
\hline \hline Facility & Date & Number of Images & $\begin{array}{c}\text { Cadence } \\
(\mathrm{s})\end{array}$ & Filter \\
\hline KeplerCam/FLWO 1.2 m & 2007 Dec 14 & 367 & 30 & Sloan $z$ band \\
Konkoly Schmidt 0.6 m & 2008 Sep 8 & 538 & 45 & $I$ band \\
Wise 0.46 m & 2008 Sep 8 & 769 & 35 & $R$ band \\
Wise 1.0 m & 2008 Sep 8 & 407 & 50 & $R$ band \\
KeplerCam/FLWO 1.2 m & 2008 Oct 19 & 350 & 33 & Sloan $i$ band \\
KeplerCam/FLWO 1.2 m & 2009 Oct 16 & 403 & 33 & Sloan $i$ band \\
\hline
\end{tabular}

Table 3

High-precision Differential Photometry of HAT-P-17

\begin{tabular}{lrccc}
\hline \hline $\begin{array}{l}\text { BJD } \\
(2,400,000+)\end{array}$ & \multicolumn{1}{c}{ Mag $^{\mathrm{a}}$} & $\sigma_{\mathrm{Mag}}$ & Mag (orig) $^{\mathrm{b}}$ & Filter \\
\hline 54449.55537 & -0.00355 & 0.00095 & 9.35242 & $z$ \\
54449.55568 & 0.00062 & 0.00094 & 9.35614 & $z$ \\
54449.55603 & -0.00172 & 0.00095 & 9.35430 & $z$ \\
54449.55633 & 0.00205 & 0.00095 & 9.35788 & $z$ \\
54449.55668 & -0.00600 & 0.00093 & 9.35026 & $z$ \\
54449.55700 & 0.00175 & 0.00095 & 9.35703 & $z$ \\
54449.55732 & -0.00625 & 0.00093 & 9.34882 & $z$ \\
54449.55768 & -0.00181 & 0.00094 & 9.35300 & $z$ \\
54449.55799 & -0.00084 & 0.00094 & 9.35409 & $z$ \\
54449.55835 & 0.00142 & 0.00093 & 9.35711 & $z$ \\
\hline
\end{tabular}

Notes.

a The out-of-transit level has been subtracted. These magnitudes have been subjected to the EPD and TFA procedures, carried out simultaneously with the transit fit.

${ }^{b}$ Raw magnitude values without application of the EPD and TFA procedures.

(This table is available in its entirety in a machine-readable form in the online journal. A portion is shown here for guidance regarding its form and content.)

RVs and the bisector variations is insignificant. Therefore, we conclude that the velocity variations are real, and that the star is orbited by a close-in giant planet.

\subsection{Global Modeling of the Data}

This section describes the procedure we followed to model the HATNet photometry, the follow-up photometry, and the RVs simultaneously. Our model for the follow-up light curves used analytic formulae based on Mandel \& Agol (2002) for the eclipse of a star by a planet, with limb darkening being prescribed by a quadratic law. The limb-darkening coefficients for the $I, R$ and Sloan $z$ and $i$ bands were interpolated from the tables by Claret (2004) for the spectroscopic parameters of the star as determined from the SME analysis (Section 3.1). The transit shape was parameterized by the normalized planetary radius $p \equiv R_{p} / R_{\star}$, the square of the impact parameter $b^{2}$, and the reciprocal of the half-duration of the transit $\zeta / R_{\star}$. We chose these parameters because of their simple geometric meanings and their negligible correlations with each other (see Bakos et al. 2010b). The relation between $\zeta / R_{\star}$ and the quantity $a / R_{\star}$, used in Section 3.1, is given by

$$
a / R_{\star}=P / 2 \pi\left(\zeta / R_{\star}\right) \sqrt{1-b^{2}} \sqrt{1-e^{2}} /(1+e \sin \omega)
$$

(see, e.g., Tingley \& Sackett 2005). Our model for the HATNet data was the simplified "P1P3" version of the Mandel \& Agol (2002) analytic functions (an expansion in terms of Legendre polynomials), for the reasons described in Bakos et al. (2010b).
Table 4

Stellar Parameters for HAT-P-17

\begin{tabular}{lcc}
\hline \hline Parameter & Value & Source \\
\hline Spectroscopic properties & & \\
$T_{\text {eff }}(\mathrm{K})$ & $5246 \pm 80$ & $\mathrm{SME}^{\mathrm{a}}$ \\
{$[\mathrm{Fe} / \mathrm{H}]$} & $0.00 \pm 0.08$ & $\mathrm{SME}$ \\
$v \sin i\left(\mathrm{~km} \mathrm{~s}^{-1}\right)$ & $0.3 \pm 0.5$ & $\mathrm{SME}$ \\
$v_{\text {mac }}\left(\mathrm{km} \mathrm{s}^{-1}\right)$ & 3.21 & $\mathrm{SME}$ \\
$v_{\text {mic }}\left(\mathrm{km} \mathrm{s}^{-1}\right)$ & 0.85 & $\mathrm{SME}$ \\
$\gamma_{\mathrm{RV}}\left(\mathrm{km} \mathrm{s}^{-1}\right)$ & $20.13 \pm 0.21$ & $\mathrm{DS}$ \\
Photometric properties & & \\
$V(\mathrm{mag})$ & 10.54 & $\mathrm{TASS}$ \\
$V-I_{C}(\mathrm{mag})$ & $0.901 \pm 0.10$ & $\mathrm{TASS}$ \\
$J(\mathrm{mag})$ & $9.017 \pm 0.022$ & $2 \mathrm{MASS}$ \\
$H(\mathrm{mag})$ & $8.619 \pm 0.029$ & $2 \mathrm{MASS}$ \\
$K_{s}(\mathrm{mag})$ & $8.544 \pm 0.025$ & $2 \mathrm{MASS}$ \\
Derived properties & & \\
$M_{\star}\left(M_{\odot}\right)$ & $0.857 \pm 0.039$ & $\mathrm{YY}+a / R_{\star}+\mathrm{SME}$ \\
$R_{\star}\left(R_{\odot}\right)$ & $0.838 \pm 0.021$ & $\mathrm{YY}+a / R_{\star}+\mathrm{SME}$ \\
$\log g_{\star}(\mathrm{cgs})$ & $4.52 \pm 0.02$ & $\mathrm{YY}+a / R_{\star}+\mathrm{SME}$ \\
$L_{\star}\left(L_{\odot}\right)$ & $0.48 \pm 0.04$ & $\mathrm{YY}+a / R_{\star}+\mathrm{SME}$ \\
$M_{V}(\mathrm{mag})$ & $5.75 \pm 0.12$ & $\mathrm{YY}+a / R_{\star}+\mathrm{SME}$ \\
$M_{K}(\mathrm{mag}, \mathrm{ESO})$ & $3.79 \pm 0.07$ & $\mathrm{YY}+a / R_{\star}+\mathrm{SME}$ \\
Age $(\mathrm{Gyr})$ & $7.8 \pm 3.3$ & $\mathrm{YY}+a / R_{\star}+\mathrm{SME}$ \\
Distance $(\mathrm{pc})$ & $90 \pm 3$ & $\mathrm{YY}+a / R_{\star}+\mathrm{SME}$ \\
\hline & &
\end{tabular}

Notes.

a SME: "Spectroscopy Made Easy" package for the analysis of high-resolution spectra (Valenti \& Piskunov 1996). These parameters rely primarily on SME, but have a small dependence also on the iterative analysis incorporating the isochrone search and global modeling of the data, as described in the text.

${ }^{\mathrm{b}} \mathrm{YY}+a / R_{\star}+\mathrm{SME}=$ based on the $\mathrm{YY}$ isochrones (Yi et al. 2001), $a / R_{\star}$ as a luminosity indicator, and the SME results.

Initial modeling of the RV observations showed deviations from a Keplerian fit highly suggestive of a second body in the system with a much longer period than the transiting planet. Thus, in our global modeling, the RV curve was parameterized by the combination of an eccentric Keplerian orbit for the inner planet with semi-amplitude $K$, and Lagrangian orbital elements $(k, h) \equiv e \times(\cos \omega, \sin \omega)$, plus an eccentric Keplerian orbit for the outer object with $K_{2}, k_{2}$ and $h_{2}$, and a systemic RV zeropoint $\gamma$ (see also Bakos et al. 2010b). Throughout this paper the subscripts " 1 " and " 2 " refer to HAT-P-17b and HAT-P-17c, respectively. If the subscript is omitted, we refer to HAT-P-17b.

We assumed a strict periodicity in the individual transit times. We assigned the transit number $N_{\text {tr }}=0$ to the last complete follow-up light curve gathered on 2009 October 16. The adjustable parameters in the fit that determine the ephemeris were chosen to be the time of the first transit center observed with HATNet, $T_{c,-65}$, and that of the last transit center observed with the FLWO $1.2 \mathrm{~m}$ telescope, $T_{c, 0}$. We used these as opposed 
to period and reference epoch in order to minimize correlations between parameters (see Pál et al. 2008). Times of midtransit for intermediate events were interpolated using these two epochs and the corresponding transit number of each event, $N_{\mathrm{tr}}$. The 11 main parameters describing the physical model were thus $T_{c,-65}, T_{c, 0}, R_{p} / R_{\star}, b^{2}, \zeta / R_{\star}, K, k \equiv e \cos \omega, h \equiv e \sin \omega, K_{2}$, $k_{2}$, and $h_{2}$. Five additional parameters were included that have to do with the instrumental configuration. These are the HATNet blend factor $B_{\text {inst, } 247}$, and $B_{\text {inst, } 248}$ which accounts for possible dilution of the transit in the 247 and 248 HATNet light curves from background stars due to the broad point-spread function $\left(20^{\prime \prime}\right.$ FWHM), the HATNet out-of-transit magnitudes $M_{0, \text { HATNet,247, }}$, and $M_{0, \text { HATNet,248, }}$, and the relative zero-point $\gamma_{\text {rel }}$ of the Keck RVs.

We extended our physical model with an instrumental model that describes brightness variations caused by systematic errors in the measurements. This was done in a similar fashion to the analysis presented by Bakos et al. (2010b). The HATNet photometry has already been EPD- and TFA-corrected before the global modeling, so we only considered corrections for systematics in the follow-up light curves. We chose the "ELTG" method, i.e., EPD was performed in "local" mode with EPD coefficients defined for each night, and TFA was performed in "global" mode using the same set of stars and TFA coefficients for all nights. The total number of fitted parameters was 16 (physical model with five configuration-related parameters) + 36 $($ local EPD $)+10($ global TFA $)=67$, i.e., much smaller than the number of data points (2866, counting only RV measurements and follow-up photometry measurements).

The joint fit was performed as described in Bakos et al. (2010b). We minimized $\chi^{2}$ in the space of parameters using a hybrid algorithm, combining the downhill simplex method (AMOEBA; see Press et al. 1992) with a classical linear leastsquares algorithm. Parameter uncertainties were derived applying the Markov chain Monte Carlo method (MCMC; see Ford 2006) using "Hyperplane-CLLS" chains (Bakos et al. 2010b). This provided the full a posteriori probability distributions of all adjusted variables. The a priori distributions of the parameters for these chains were chosen to be Gaussian, with eigenvalues and eigenvectors derived from the Fisher covariance matrix for the best-fit solution. The Fisher covariance matrix was calculated analytically using the partial derivatives given by Pál (2009a).

Following this procedure we obtained a posteriori distributions for all fitted variables, and other quantities of interest such as $a / R_{\star}$. As described in Section 3.1, $a / R_{\star}$ was used with stellar evolution models to infer a theoretical value for $\log g_{\star}$ that is significantly more accurate than the spectroscopic value. The improved estimate was in turn applied to a second iteration of the SME analysis, as explained previously, to obtain better estimates of $T_{\text {eff } \star}$ and $[\mathrm{Fe} / \mathrm{H}]$. The global modeling was then repeated with updated limb-darkening coefficients based on those new spectroscopic determinations. The resulting geometric parameters pertaining to the light curves and velocity curves are listed in Tables 5 and 6. All quantities in these tables, except for the limb-darkening coefficients, are the product of global modeling.

Included in Table 5 is the RV “jitter." This quantity accounts for RV variability due to rotational modulation of stellar surface features, stellar pulsation, undetected planets, and uncorrected systematic errors in the velocity reduction (Wright 2005). We adopted a jitter value of $2.0 \mathrm{~m} \mathrm{~s}^{-1}$ and added it in quadrature to the measurement uncertainties in Table 1 . This jitter value
Table 5

Orbital and Planetary Parameters for HAT-P-17b

\begin{tabular}{|c|c|}
\hline Parameter & Value \\
\hline \multicolumn{2}{|l|}{ Light curve parameters } \\
\hline$P$ (days) & $10.338523 \pm 0.000009$ \\
\hline$T_{c}(\mathrm{BJD})^{\mathrm{a}}$ & $2454801.16943 \pm 0.00020$ \\
\hline$T_{14}(\text { days })^{\mathrm{a}}$ & $0.1690 \pm 0.0009$ \\
\hline$T_{12}=T_{34}(\text { days })^{\mathrm{a}}$ & $0.0204 \pm 0.0009$ \\
\hline$a / R_{\star}$ & $22.63 \pm 0.49$ \\
\hline$\zeta / R_{\star}$ & $13.44 \pm 0.04$ \\
\hline$R_{p} / R_{\star}$ & $0.1238 \pm 0.0010$ \\
\hline$b^{2}$ & $0.097_{-0.034}^{+0.031}$ \\
\hline$b \equiv a \cos i / R_{\star}$ & $0.311_{-0.067}^{+0.045}$ \\
\hline$i(\operatorname{deg})$ & $89.2_{-0.1}^{+0.2}$ \\
\hline$T_{\text {peri }}$ (days) & $2454803.25 \pm 0.05$ \\
\hline \multicolumn{2}{|c|}{ Limb-darkening coefficients ${ }^{b}$} \\
\hline$c_{1}, i$ (linear term) & 0.3592 \\
\hline$c_{2}, i$ (quadratic term) & 0.2759 \\
\hline$c_{1}, z$ & 0.2843 \\
\hline$c_{2}, z$ & 0.2882 \\
\hline$c_{1}, I$ & 0.3347 \\
\hline$c_{2}, I$ & 0.2800 \\
\hline \multicolumn{2}{|l|}{ RV parameters } \\
\hline$K\left(\mathrm{~m} \mathrm{~s}^{-1}\right)$ & $58.8 \pm 0.9$ \\
\hline$k_{\mathrm{RV}}^{\mathrm{c}}$ & $-0.318 \pm 0.005$ \\
\hline$h_{\mathrm{RV}}^{\mathrm{c}}$ & $-0.127 \pm 0.012$ \\
\hline$e$ & $0.342 \pm 0.006$ \\
\hline$\omega(\operatorname{deg})$ & $201 \pm 1$ \\
\hline $\mathrm{RV}$ jitter $\left(\mathrm{m} \mathrm{s}^{-1}\right)$ & 2.0 \\
\hline \multicolumn{2}{|c|}{ Secondary eclipse parameters } \\
\hline$T_{s}(\mathrm{BJD})$ & $2454804.268 \pm 0.030$ \\
\hline$T_{s, 14}$ & $0.1331 \pm 0.0034$ \\
\hline$T_{s, 12}$ & $0.0155 \pm 0.0007$ \\
\hline \multicolumn{2}{|l|}{ Planetary parameters } \\
\hline$M_{p}\left(M_{\mathrm{J}}\right)$ & $0.534 \pm 0.018$ \\
\hline$R_{p}\left(R_{\mathrm{J}}\right)$ & $1.010 \pm 0.029$ \\
\hline$C\left(M_{p}, R_{p}\right)^{\mathrm{d}}$ & 0.45 \\
\hline$\rho_{p}\left(\mathrm{~g} \mathrm{~cm}^{-3}\right)$ & $0.64 \pm 0.05$ \\
\hline $\log g_{p}(\operatorname{cgs})$ & $3.11 \pm 0.02$ \\
\hline$a(\mathrm{AU})$ & $0.0882 \pm 0.0014$ \\
\hline$T_{\text {eq }}(\mathrm{K})$ & $792 \pm 15$ \\
\hline$\Theta^{\mathrm{e}}$ & $0.109 \pm 0.004$ \\
\hline$F_{\text {per }}\left(10^{8} \mathrm{erg} \mathrm{s}^{-1} \mathrm{~cm}^{-2}\right)^{\mathrm{f}}$ & $1.93 \pm 0.14$ \\
\hline$F_{\text {ap }}\left(10^{7} \mathrm{erg} \mathrm{s}^{-1} \mathrm{~cm}^{-2}\right)^{\mathrm{f}}$ & $4.64 \pm 0.36$ \\
\hline$\langle F\rangle\left(10^{7} \mathrm{erg} \mathrm{s}^{-1} \mathrm{~cm}^{-2}\right)^{\mathrm{f}}$ & $8.89 \pm 0.67$ \\
\hline
\end{tabular}

Notes.

a $T_{c}$ : reference epoch of mid-transit that minimizes the correlation with the orbital period. It corresponds to $N_{\mathrm{tr}}=-32$. $T_{14}$ : total transit duration, time between first to last contact; $T_{12}=T_{34}$ : ingress/egress time, time between first and second, or third and fourth contact.

b Values for a quadratic law, adopted from the tabulations by Claret (2004) according to the spectroscopic (SME) parameters listed in Table 4.

${ }^{\mathrm{c}}$ Lagrangian orbital parameters derived from the global modeling, and primarily determined by the RV data.

${ }^{\mathrm{d}}$ Correlation coefficient between the planetary mass $M_{p}$ and radius $R_{p}$.

e The Safronov number is given by $\Theta=\frac{1}{2}\left(V_{\text {esc }} / V_{\text {orb }}\right)^{2}=\left(a / R_{p}\right)\left(M_{p} / M_{\star}\right)$ (see Hansen \& Barman 2007).

${ }^{f}$ Incoming flux per unit surface area, averaged over the orbit.

was chosen so that reduced $\chi^{2}=1$ for the RV data of the global fit. This value is consistent with the jitter of an ensemble of chromospherically quiet, late G/early $\mathrm{K}$ dwarf stars (Wright 2005) and the jitter adopted so that $\chi^{2}=1$ for recent low-mass planet detections by Keck/HIRES (Howard 
Table 6

Orbital and Planetary Parameters for HAT-P-17c

\begin{tabular}{|c|c|}
\hline Parameter & Value \\
\hline \multicolumn{2}{|c|}{ RV parameters, as induced by HAT-P-17c } \\
\hline$P_{2}$ (days) & $1610 \pm 20$ \\
\hline$T_{2 c}{ }^{\mathrm{a}}(\mathrm{BJD})$ & $2452820.0 \pm 17.7$ \\
\hline$K_{2}\left(\mathrm{~m} \mathrm{~s}^{-1}\right)$ & $25.2_{-2.5}^{+3.5}$ \\
\hline$k_{2}$ & $0.076_{-0.09}^{+0.10}$ \\
\hline$h_{2}$ & $-0.007 \pm 0.076$ \\
\hline$e_{2}$ & $0.086_{-0.067}^{+0.099}$ \\
\hline$\omega_{2}$ & $286^{\circ} \pm 179^{\circ}$ \\
\hline$T_{2, \text { peri }}$ (days) & $2452456.35_{-176.55}^{+335.66}$ \\
\hline \multicolumn{2}{|c|}{ Hypothetical light curve parameters, HAT-P-17 $\mathrm{c}^{\mathrm{b}}$} \\
\hline$T_{2,14^{\mathrm{c}}}$ (days) & $0.881 \pm 0.039$ \\
\hline$T_{2,12}=T_{34}$ (days) & $0.0950 \pm 0.0037$ \\
\hline \multicolumn{2}{|c|}{ Hypothetical secondary eclipse parameters for HAT-P-17 $\mathrm{c}^{\mathrm{a}}$} \\
\hline$T_{2 s}(\mathrm{BJD})$ & $2453703.3 \pm 36.4$ \\
\hline$T_{2 s, 14}$ (days) & $0.869 \pm 0.037$ \\
\hline$T_{2 s, 12}$ (days) & $0.0950 \pm 0.0037$ \\
\hline \multicolumn{2}{|c|}{ Planetary parameters for HAT-P-17c } \\
\hline$m_{2} \sin i_{2}\left(M_{\mathrm{J}}\right)$ & $1.31_{-0.15}^{+0.18}$ \\
\hline$a_{2}(\mathrm{AU})$ & $2.555 \pm 0.041$ \\
\hline$T_{2, \mathrm{eq}}(\mathrm{K})$ & $145 \pm 3$ \\
\hline$F_{2, \text { per }}\left(10^{5} \mathrm{erg} \mathrm{s}^{-1} \mathrm{~cm}^{-2}\right) \mathrm{d}$ & $1.2 \pm 0.156$ \\
\hline$F_{2, \text { ap }}\left(10^{4} \mathrm{erg} \mathrm{s}^{-1} \mathrm{~cm}^{-2}\right)^{\mathrm{d}}$ & $8.41 \pm 0.86$ \\
\hline$\left\langle F_{2}\right\rangle\left(10^{5} \mathrm{erg} \mathrm{s}^{-1} \mathrm{~cm}^{-2}\right)^{\mathrm{d}}$ & $1 \pm 0.0762$ \\
\hline
\end{tabular}

Notes.

a $T_{2 c}$ would be the center of transit of HAT-P-17c, if its (unknown) inclination is $90^{\circ}$.

b Transits of HAT-P-17c have not been observed. The values are for guidance only, and assume zero impact parameter.

c $T_{14}$ : total transit duration, time between first to last contact, assuming zero impact parameter. $T_{12}=T_{34}$ : ingress/egress time, time between first and second, or third and fourth contact. Note that these values are hypothetical, and transits of HAT-P-17c have not been observed.

${ }^{\mathrm{d}}$ Incoming flux per unit surface area in periastron, apastron, and averaged over the orbit.

et al. 2009, 2011a, 2011b). Auxiliary parameters not listed in the tables are $T_{\mathrm{c},-65}=2454449.65966 \pm 0.00036$ (BJD), $T_{\mathrm{c}, 0}=2455121.66363 \pm 0.00034$ (BJD), the blending factors $B_{\text {instr,247 }}=0.87 \pm 0.04$ and $B_{\text {instr,248 }}=0.69 \pm 0.16$, and $\gamma_{\text {rel }}=25.1 \pm 1.4 \mathrm{~ms}^{-1}$. The latter quantity represents an arbitrary offset for the Keck RVs, and does not correspond to the true center-of-mass velocity of the system, which was listed earlier in Table $4\left(\gamma_{\mathrm{RV}}\right)$.

The planetary parameters and their uncertainties are derived from the a posteriori distributions of the stellar, light curve, and RV parameters. We find an inner planet mass of $M_{p}=$ $0.534 \pm 0.018 M_{\mathrm{J}}$ and a radius of $R_{p}=1.010 \pm 0.029 R_{\mathrm{J}}$, giving a mean density $\rho_{p}=0.64 \pm 0.05 \mathrm{~g} \mathrm{~cm}^{-3}$. These and other planetary parameters are listed at the bottom of Table 5. We note that the inner planet's eccentricity is significantly nonzero: $e=0.342 \pm 0.006$ and $\omega=201^{\circ} \pm 1^{\circ}$.

We initially considered a model with a single, transiting planet but rejected this model because of the high velocity rms $\left(10.9 \mathrm{~m} \mathrm{~s}^{-1}\right)$ and velocity residuals that are coherent in time. These residuals suggest a second (non-transiting) planet with a substantially longer orbital period. The two-planet model present here significantly improves on our initial one-planet model and has a velocity rms of $3.07 \mathrm{~m} \mathrm{~s}^{-1}$. HAT-P-17c is a long-period jovian planet with a minimum mass $m_{2} \sin i_{2}=$
$1.31_{-0.15}^{+0.18} M_{\mathrm{J}}$ and orbital period $P_{2}=1610 \pm 20$ days. Its eccentricity of $e_{2}=0.086_{-0.067}^{+0.099}$ is consistent with a circular orbit. Because we have only measured about half of an orbit of HAT-P-17c, we conservatively adopt $95.4 \%$ confidence intervals (" $2 \sigma$ errors") for the error estimates on parameters associated with this planet. (Unless noted, all other parameter uncertainties in this paper are $68.3 \%$ confidence intervals, " $1 \sigma$ errors.") Figure 5 shows the distributions of and correlations between $m_{2} \sin i_{2}, P_{2}$, and $e_{2}$ from the MCMC analysis. Correlations between the Lagrangian orbital parameters $k_{2}=e_{2} \cos \omega_{2}$ and $h_{2}=e_{2} \sin \omega_{2}$ are also shown.

We caution that even with the more conservative $2 \sigma$ errors, the parameter uncertainties for HAT-P-17c may still be underestimated. (The parameters describing HAT-P-17b are robust to changes in parameters describing HAT-P-17c.) This additional, difficult-to-quantify uncertainty could come from additional long-period planets in the system whose RV signatures conspire to produce the signal we identify as HAT-P-17c. To check whether the errors are Gaussian distributed, an implicit assumption in the MCMC analysis, we examined the distribution of RV residuals to the two-planet model. This distribution is well approximated by a Gaussian with a width comparable to the rms of $3.07 \mathrm{~m} \mathrm{~s}^{-1}$, suggesting that the assumption of Gaussianity, implicit in the MCMC analysis, is justified. An analysis of additional RV measurements will be most fruitful when HAT-P-17c has completed one orbit, currently forecast for mid-2012.

\section{DISCUSSION}

We present the detection of HAT-P-17b, a transiting hot Saturn in an eccentric orbit, and HAT-P-17c, a cold Jupiter with an unknown orbital inclination. In this section, we discuss these two planets in the context of recent models and trends, the statistics of nearly 100 TEPs, and the small number of multiplanet systems with one or more transiting members.

\subsection{The Planet HAT-P-17b}

As seen in Figure 6, HAT-P-17b has a radius that is typical of other known TEPs with masses in the range 0.5-0.6 $M_{\mathrm{J}}$. Comparing HAT-P-17b to the theoretical models by Fortney et al. (2007), we find that it is consistent with gas-dominated planet having a core mass of $M_{C} \sim 25 M_{\oplus}$ for an age of $4 \mathrm{Gyr}$, or somewhat less than this for older ages. HAT-P-17b is not inflated relative to theoretical models. This lack of inflation is consistent with the relatively cool temperature $\left(T_{\text {eq }}=792 \pm 15 \mathrm{~K}\right)$ of HAT-P-17b.

\subsubsection{Spin-Orbit Alignment}

In the core accretion theory of planet formation, hot giant planets like HAT-P-17b form beyond the ice line (a few AU from the host star) and subsequently migrate inward. Several migration mechanisms have been proposed. Tidal interactions with the protoplanetary disk (Lin et al. 1996) deliver gas giants with uniformly low obliquity. Alternatively, Kozai cycles (Fabrycky \& Tremaine 2007) or planet-planet scattering (Chatterjee et al. 2009) leave the migrated planets in highobliquity orbits, possibly with high eccentricity (depending on the degree of tidal damping). Both high- and low-obliquity systems have been observed by the Rossiter-McLaughlin (R-M) effect, suggesting some combination of migration mechanisms (Fabrycky \& Winn 2009; Morton \& Johnson 2010; Triaud et al. 2010). 

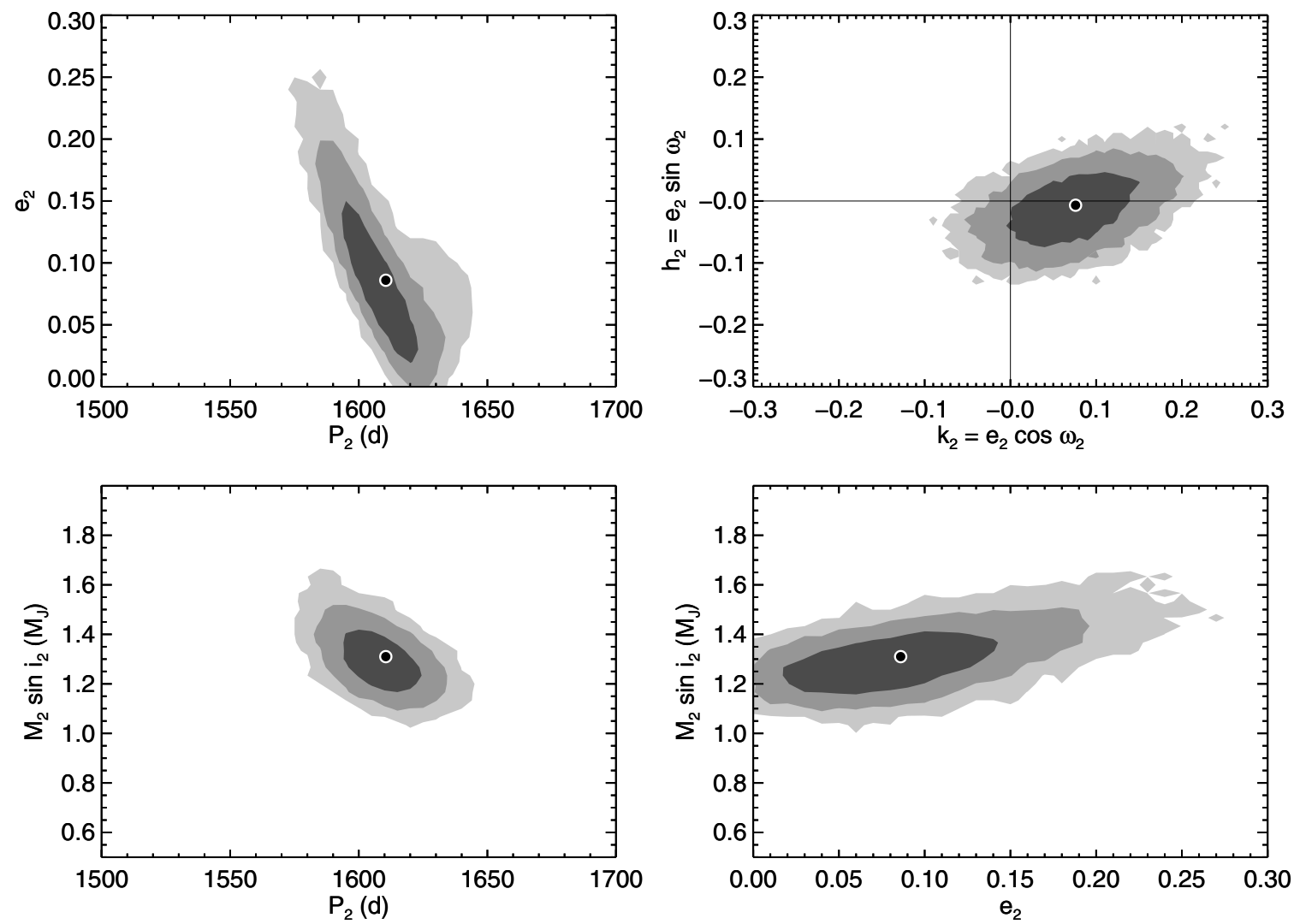

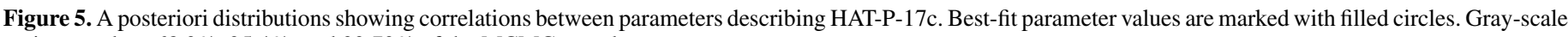
regions enclose $68.3 \%, 95.4 \%$, and $99.73 \%$ of the MCMC samples.

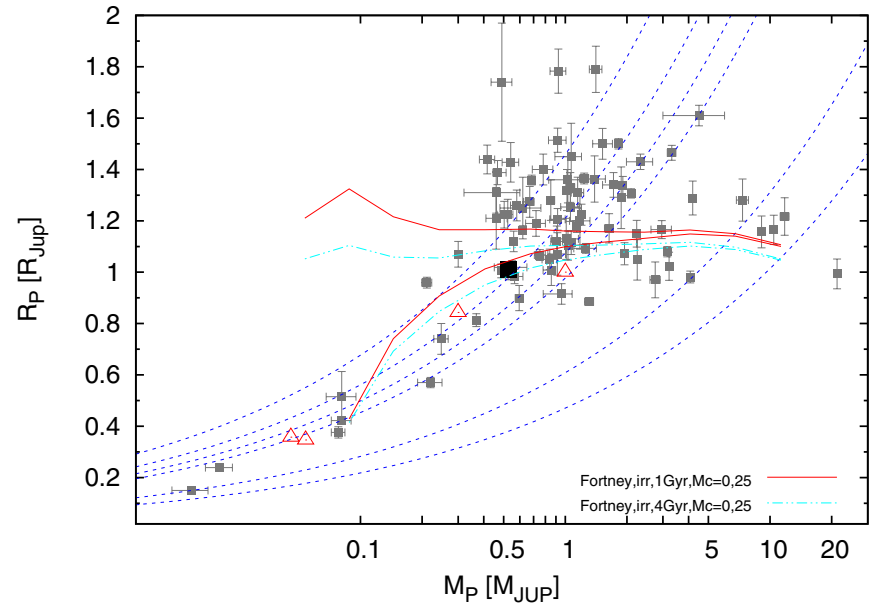

Figure 6. Mass-radius diagram of known TEPs (small filled squares). HAT-P$17 \mathrm{~b}$ is shown as a large filled square. Overlaid are Fortney et al. (2007) planetary isochrones interpolated to the solar equivalent semimajor axis of HAT-P-17b for ages of $1.0 \mathrm{Gyr}$ (upper solid lines) and $4 \mathrm{Gyr}$ (lower dash-dotted lines) and core masses of 0 and $25 M_{\oplus}$ (upper and lower lines, respectively), as well as isodensity lines for $0.4,0.7,1.0,1.33,5.5$, and $11.9 \mathrm{~g} \mathrm{~cm}^{-3}$ (dashed lines). Solar system planets are shown with open triangles.

(A color version of this figure is available in the online journal.)

Winn et al. (2010) recently noted that nearly all misaligned (high-obliquity) planets orbit hot stars $\left(T_{\text {eff }}>6250 \mathrm{~K}\right)$. They suggested that all hot giant planets migrated by one of the high-obliquity mechanisms and that planets orbiting cool stars subsequently align the spin axis of the convective zones and photospheres of their hosts with the orbital plane. Stars above this threshold temperature lack a significant convective zone and their close-in giant planets remain in high-obliquity orbits.

Although HAT-P-17 is a cool star, the Winn et al. model predicts spin-orbit misalignment because the wider, eccentric orbit of HAT-P-17b lengthens the timescale for orbital decay considerably. Of the known TEPs, HAT-P-17b has the longest expected timescale except for HD 80606b (J. Winn 2010, private communication). In particular, HAT-P-17b has a longer timescale than WASP-8b, which is known to be misaligned (Queloz et al. $2010)$. This planet ( $P=8$ days, $e=0.31$ ) is broadly similar to HAT-P-17b and also orbits a relatively cool star $\left(T_{\text {eff }}=5600 \mathrm{~K}\right)$.

Measuring the projected spin-orbit angle $\lambda$ of HAT-P-17b is a challenging but plausible proposition with HIRES. We estimate a $4-11 \mathrm{~m} \mathrm{~s}^{-1}$ amplitude R-M effect for $v \sin i=0.3-0.8 \mathrm{~km} \mathrm{~s}^{-1}$.

\subsubsection{Similarity to HAT-P-15b}

HAT-P-17b is strikingly similar to HAT-P-15b (Kovács et al. 2010) in orbital period (10.3 days and 10.9 days, respectively) and eccentricity ( 0.35 and 0.19 , respectively). The mass of HAT-P-15b is significantly larger though $\left(0.5 M_{\mathrm{J}}\right.$ and $1.9 M_{\mathrm{J}}$, respectively). These two planets are the only ground-based transit discoveries with orbital periods longer than 10 days. (Other transiting planets with $P>10$ days include those detected by space-based transit surveys and two planets discovered by RVs that were later shown to transit.) The bias toward detecting planets with short orbital periods with ground-based transit searches stems from the observational window function of a longitudinally spaced multi-site telescope network (von Braun et al. 2009). The HAT-South survey will significantly improve the detection of longer period transiting planets with a $50 \%$ detection rate out to orbital periods of 12 days (Bakos et al. 2009). 


\subsection{Transit Timing Variations}

The presence of a second detected planet in the HAT-P-17 system raises the possibility of transit timing variations (TTVs; Holman \& Murray 2005; Agol et al. 2005). However, because HAT-P-17b and HAT-P-17c are widely separated $\left(a_{2} / a_{1} \sim 31\right)$ and HAT-P-17c is on a nearly circular orbit, the two planets interact very weakly. The TTVs between successive orbits are expected to be less than $1 \mathrm{~s}$, undetectable with current techniques. However, over the peak-to-trough half-cycle of the orbit of HAT-P-17c deviations from a linear ephemeris will be in the range 4-8 s, depending on the relative inclination of the two planets and the relative phase of dynamical variations compared to light travel time variations.

\subsection{The Planet HAT-P-17c}

HAT-P-17c is an approximately Jupiter-mass planet separated from its host star by about half the Sun-Jupiter separation. Despite having only observed $50 \%$ of the orbit of HAT-P-17c, its orbital parameters are well constrained by the model of a long-period planet (Figure 5). While we cannot completely rule out a highly eccentric orbit, only $3 \%$ of the MCMC samples have $e>0.3$.

The 2007 December 14 light curve (Figure 3) showing a partial transit of HAT-P-17b is during the broad transit window of HAT-P-17c. We interpret the detected transit as due to HAT-P-17b because the timing precisely matches the ephemeris derived from other transits. We do not detect additional transits (possibly due to HAT-P-17c) in that light curve. Because that light curve is the only one taken in $z$ band we cannot compare the photometric level of this transit with others to see if it was taken entirely when HAT-P-17c was in transit.

Based on the current orbital fit, the next opportunity to search for a transit of HAT-P-17c is in 2012 October. The timing is favorable for an observing campaign as the star is visible for $\sim 5 \mathrm{hr}$ per night from mid-northern latitudes. From the ground, a coordinated, multi-site search spanning a range of longitudes is likely necessary to rule in or out $\sim 1 \%$ deep transits of maximum duration 0.881 days. The a priori transit probability for HAT-P- $17 \mathrm{c}$ is $0.15 \%$, although if HAT-P-17b and HAT-P-17c have mutual inclination of $\leqslant 5^{\circ}$, then the transit probability is $1.7 \%$.

\subsection{Planet Multiplicity}

The migration mechanism of hot jovian planets remains a major outstanding problem of planet formation and evolution. The presence of additional massive planets in a system points to migration within the protoplanetary disk, while the absence of additional planets suggests a more disruptive mechanism such as planet-planet scattering or the Kozai mechanism.

Wright et al. (2009) measured the rate of planet multiplicity and found that $14 \%$ of exoplanet host stars are multi-planet systems and another 14\% show evidence of multiplicity in the form of an RV trend. Here, we compute the fraction of stars hosting a "cool jovian planet" $\left(m \sin i>0.2 M_{\mathrm{J}}\right.$ and $a>0.2 \mathrm{AU})$ that also host a "hot jovian planet" $(m \sin i>$ $0.2 M_{\mathrm{J}}$ and $\left.a<0.2 \mathrm{AU}\right)$. We used the Exoplanet Orbit Database $^{13}$ (Wright et al. 2011) of planets with well-defined orbital parameters. Of the 375 planet hosts (including HAT-P17), we find 106 stars that host a hot jovian planet and 204 stars that host one or more cool jovian planets. Of the latter

\footnotetext{
13 http://exoplanets.org
}

group, 10 stars (5\%) also host a hot jovian planet. Restricting the hot jovian planets to $a<0.1 \mathrm{AU}, 6 / 204=3 \%$ of stars host both cool and hot jovian planets. Note that this selection of planets does not suffer from a significant detection bias; the Doppler signal from a hot jovian planet is essentially always detectable for systems with a detected cool jovian planet. While hot jovian planets represent a disproportionally large fraction of the known planets due to observational selection effects, multiplanet systems like HAT-P-17 are rare.

The current orbital parameters of HAT-P-17b,c present a challenge for planet formation and migration scenarios. If both planets formed at several $\mathrm{AU}$, how did HAT-P-17b migrate to its current close-in orbit and acquire a substantial eccentricity, while HAT-P-17c remained in the outer part of the system with an apparently low eccentricity orbit? Answering these questions will require a full characterization of HAT-P-17c (at least one complete orbit) and perhaps a more thorough search for additional planets in other hot Jupiter systems. We point out that in models of our solar system, the final architecture often depends on a sequence of contingent events in the growth and migration history. In the Nice model (Morbidelli 2011), Saturn quickly grew to gas giant size and migrated into resonance with Jupiter. Had the growth and initial migration of Saturn occurred later, it might have been dynamically decoupled from Jupiter, perhaps resulting in a qualitatively different final architecture for our entire solar system.

We thank E. Agol, H. Knutson, J. Winn, and J. Wright for helpful conversations. HATNet operations have been funded by NASA grants NNG04GN74G, NNX08AF23G, and SAO IR\&D grants. A.W.H. gratefully acknowledges support from a Townes Post-doctoral Fellowship at the U. C. Berkeley Space Sciences Laboratory. Work of G.Á.B. and J. Johnson were supported by the Postdoctoral Fellowship of the NSF Astronomy and Astrophysics Program (AST-0702843 and AST-0702821, respectively). G.T. acknowledges partial support from NASA grant NNX09AF59G. We acknowledge partial support also from the Kepler mission under NASA Cooperative Agreement NCC2-1390 (D.W.L.: PI). G.K. thanks the Hungarian Scientific Research Foundation (OTKA) for support through grant K-81373. T.M. acknowledges the Israel Science Foundation (grant 655/07). This research has made use of Keck telescope time granted through NOAO and NASA. We thank Ezra Mashal for his help in operating the Wise-HAT telescope over the past years. We thank the TLC project (M. Holman and J. Winn) for swapping time on the $1.2 \mathrm{~m}$ telescope at short notice. This research has made use of the Exoplanet Orbit Database and the Exoplanet Data Explorer at exoplanets.org, the SIMBAD database (operated at CDS, Strasbourg, France), and NASA's Astrophysics Data System Bibliographic Services. Finally, the authors wish to extend special thanks to those of Hawaiian ancestry on whose sacred mountain of Mauna Kea we were privileged to be guests. Without their generous hospitality, the Keck observations presented herein would not have been possible.

\section{REFERENCES}

Agol, E., Steffen, J., Sari, R., \& Clarkson, W. 2005, MNRAS, 359, 567

Bakos, G. Á., Afonso, C., Henning, T., et al. 2009, in IAU Symp. 253, Transiting Planets, ed. F. Pont, D. D. Sasselov, \& M. J. Holman (Cambridge: Cambridge Univ. Press), 354

Bakos, G. Á., Howard, A. W., Noyes, R. W., et al. 2010a, ApJ, 707, 446

Bakos, G. Á., Kovács, G., Torres, G., et al. 2007, ApJ, 670, 826

Bakos, G. Á., Noyes, R. W., Kovács, G., et al. 2004, PASP, 116, 266

Bakos, G. Á., Torres, G., Pál, A., et al. 2010b, ApJ, 710, 1724 
Batalha, N. M. 2010, ApJ, 713, L109

Borucki, W. J., Koch, D. G., Basri, G., et al. 2011, ApJ, 728, 117

Bouchy, F., Udry, S., Mayor, M., et al. 2005, A\&A, 444, L15

Butler, R. P., Marcy, G. W., Williams, E., et al. 1996, PASP, 108, 500

Butler, R. P., Vogt, S. S., Marcy, G. W., et al. 2004, ApJ, 617, 580

Carpenter, J. M. 2001, AJ, 121, 2851

Charbonneau, D., Berta, Z. K., Irwin, J., et al. 2009, Nature, 462, 891

Charbonneau, D., Brown, T. M., Latham, D. W., \& Mayor, M. 2000, ApJ, 529 L45

Chatterjee, S., Ford, E. B., Matsumura, S., \& Rasio, F. A. 2009, ApJ, 686, 580

Claret, A. 2004, A\&A, 428, 1001

Droege, T. F., Richmond, M. W., \& Sallman, M. 2006, PASP, 118, 1666

Fabrycky, D., \& Tremaine, S. 2007, ApJ, 669, 1298

Fabrycky, D. C., \& Winn, J. N. 2009, ApJ, 696, 1230

Ford, E. 2006, ApJ, 642, 505

Fortney, J. J., Marley, M. S., \& Barnes, J. W. 2007, ApJ, 659, 1661

Gillon, M., Pont, F., Demory, B.-O., Mallmann, F., et al. 2007, A\&A, 472, L13

Hansen, B. M. S., \& Barman, T. 2007, ApJ, 671, 861

Hebb, L., Collier-Cameron, A., Loeillet, B., et al. 2009, ApJ, 693, 1920

Henry, G. W., Marcy, G. W., Butler, R. P., \& Vogt, S. S. 2000, ApJ, 529, L41

Holman, M. J., \& Murray, N. W. 2005, Science, 307, 1288

Howard, A. W., Johnson, J. A., Marcy, G. W., et al. 2009, ApJ, 696, 75

Howard, A. W., Johnson, J. A., Marcy, G. W., et al. 2011a, ApJ, 730, 10

Howard, A. W., Johnson, J. A., Marcy, G. W., et al. 2011b, ApJ, 726, 73

Isaacson, H., \& Fischer, D. A. 2010, ApJ, 725, 875

Knutson, H. A., Howard, A. W., \& Isaacson, H. 2010, ApJ, 720, 1569

Kovács, G., Bakos, G. Á., Hartman, J. D., et al. 2010, ApJ, 724, 866

Kovács, G., Bakos, G. Á., \& Noyes, R. W. 2005, MNRAS, 356, 557

Kovács, G., Zucker, S., \& Mazeh, T. 2002, A\&A, 391, 369

Latham, D. W. 1992, in IAU Coll. 135, in ASP Conf. Ser. 32, Complementary Approaches to Double and Multiple Star Research, ed. H. A. McAlister \& W. I. Hartkopf (San Francisco, CA: ASP), 110

Léger, A., Rouan, D., Schneider, J., et al. 2009, A\&A, 506, 287

Lin, D. N. C., Bodenheimer, P., \& Richardson, D. C. 1996, Nature, 380, 606

Morbidelli, A. 2011, arXiv:1106.4114
Morton, T. D., \& Johnson, J. A. 2011, ApJ, 729, 138

Pál, A. 2009a, MNRAS, 396, 1737

Pál, A. 2009b, PhD thesis, Eötvös Loránd Univ.

Pál, A., \& Bakos, G. Á. 2006, PASP, 118, 1474

Press, W. H., Teukolsky, S. A., Vetterling, W. T., \& Flannery, B. P. 1992,

Numerical Recipes in C: The Art of Scientific Computing (2nd ed.; Cambridge: Cambridge Univ. Press)

Mandel, K., \& Agol, E. 2002, ApJ, 580, L171

Marcy, G. W., \& Butler, R. P. 1992, PASP, 104, 270

Pál, A., Bakos, G. Á., Torres, G., et al. 2008, ApJ, 680, 1450

Queloz, D., Anderson, D., Collier Cameron, A., et al. 2010, A\&A, 517, 1

Queloz, D., Bouchy, F., Moutou, C., et al. 2009, A\&A, 506, 303

Shporer, A., Bakos, G. Á., Mazeh, T., Kovács, G., \& Sipöcz, B. 2009, in IAU Symp. 253, Transiting Planets, ed. F. Pont, D. D. Sasselov, \& M. J. Holman (Cambridge: Cambridge Univ. Press), 331

Skrutskie, M. F., Cutri, R. M., Stiening, R., et al. 2006, AJ, 131, 1163

Sozzetti, A., Torres, G., Charbonneau, D., et al. 2007, ApJ, 664, 1190

Steffen, J. H., Batalha, N. M., Borucki, W. J., et al. 2010, ApJ, 725, 1226

Tingley, B., \& Sackett, P. D. 2005, ApJ, 627, 1011

Torres, G., Bakos, G. Á., Kovács, G., et al. 2007, ApJ, 666, 121

Torres, G., Neuhäuser, R., \& Guenther, E. W. 2002, AJ, 123, 1701

Triaud, A. H. M. J., Collier Cameron, A., Queloz, D., et al. 2010, A\&A, 524, A25

Valenti, J. A., Butler, R. P., \& Marcy, G. W. 1995, PASP, 107, 966

Valenti, J. A., \& Fischer, D. A. 2005, ApJS, 159, 141

Valenti, J. A., \& Piskunov, N. 1996, A\&AS, 118, 595

Vaughan, A. H., Preston, G. W., \& Wilson, O. C. 1978, PASP, 90, 267

Vogt, S. S., Allen, S. L., Bigelow, B. C., et al. 1994, Proc. SPIE, 2198, 362

von Braun, K., Kane, S. R., \& Ciardi, D. R. 2009, ApJ, 702, 779

Winn, J. N., Fabrycky, D., Albrecht, S., \& Johnson, J. A. 2010, ApJ, 718, L145

Wright, J. T. 2005, PASP, 117, 657

Wright, J. T., Fakhouri, O., Marcy, G. W., et al. 2011, PASP, 123, 412

Wright, J. T., Upadhyay, S., Marcy, G. W., et al. 2009, ApJ, 693, 1084

Wu, Y., Murray, N. W., \& Ramsahai, J. M. 2007, ApJ, 670, 820

Yi, S. K., Demarque, P., Kim, Y.-C., et al. 2001, ApJS, 136, 417 